\title{
Security Requirements Engineering in the Wild: A Survey of Common Practices
}

\author{
Golnaz Elahi \\ Department of Computer Science \\ University of Toronto, Canada \\ gelahi@cs.toronto.edu
}

\author{
Eric Yu \\ Faculty of Information \\ University of Toronto, Canada \\ yu@ischool.utoronto.ca
}

\author{
Tong Li, Lin Liu \\ School of Software \\ Tsinghua University, China \\ tong,linliu@ischool.utoronto.ca
}

\begin{abstract}
Various governmental or academic institutes survey current security trends, and report vulnerabilities, security breaches, and their costs. However, it is unclear whether (and how) practitioners analyze these vulnerabilities and attacks to arrive at security requirements and decide on security solutions. What modeling methods are used for eliciting, analyzing, and documenting security requirements in real-world practice? This paper intends to answer such questions through a survey of security requirements engineering practices. 374 software professionals from 237 International and Chinese rms participated in the survey. The results show businesses often try to consider security from early stages of the development life cycle; however, ultimately, security is left to be built into the system at the implementation phase. We observed that practitioners favour qualitative risk assessment rather than quantitative approaches, and this helps them consider more varieties of factors when comparing alternative security design solutions.
\end{abstract}

Keywords-Requirements Engineering; Security Requirements; Common Security Attacks and Vulnerabilities;

\section{INTRODUCTION}

Security attacks against new services (e.g., social media) and new operating environments (e.g., mobile devices) emerge rapidly. Costs of security breaches are a huge burden on governments and businesses. Various governmental or academic institutes survey the current security trends, and report discovered vulnerability, breaches, and their costs (e.g., CERT maintains and publishes reports of discovered vulnerabilities, Open Web Application Security Project (OWASP) keeps track of top web application security risks).

Most of security-related surveys focus on trends of vulnerabilities and breaches, but security Requirements Engineering (RE) practices that are actually adopted in industrial projects are unclear. Although we have a relatively extensive knowledge about common vulnerabilities and historical attacks (e.g., NVD, CWE, NIST), the real-world approach to software security, (e.g., the modeling and analysis techniques that are used in practice) are not well studied.

Few research studies have sough for answers to these questions: How security expectations of stakeholders are involved in security RE practices? What modeling methods are used as part of the activities for eliciting, analyzing, and documenting security requirements? Do practitioners use available empirical security knowledge sources such as
National Vulnerability Database (NVD), Common Weakness Enumeration (CWE), and security standards? Do practitioners analyze vulnerabilities and attacks, evaluate their risks, and then decide on security solutions? Do practitioners rather assess risks and attacks qualitatively or quantitatively?

The answer to these questions helps understand current practices; Researchers and practitioners would learn from successful and failed methods, which helps evaluate whether the research on security $\mathrm{RE}$ has contributed to real-world practices. Such studies can lead to identifying methods and activities that are actually needed, and uncover main and immediate challenges of practitioners which demands further research focus.

This paper reports on an empirical study to answer some of the raised questions about security RE practices in the wild. The survey consists of a series of questions about security RE practices in a number of Chinese and International rms located in China. In this work, we survey how software professionals deal with security requirements and make trade-offs in practice, what are their sources of security knowledge, and what explicit security RE modeling and analysis methods they use. Based on the survey results, we develop hypotheses about current practices and methods of security requirements analysis, and extract a set of criteria for a practical and systematic security requirements modeling and analysis method.

\section{Purpose And Research Questions}

The purpose of this study is to draw an understanding of real-world security RE practices. This study intends to discover practical challenges, which help us form hypotheses about missing or inef cient security RE methods. The goals of this study are to discover:

- The development stage that security concerns are considered.

- The approach to security requirements elicitation, modeling, and documentation.

- Security knowledge sources and standards that are exercised by software professionals.

- The approach to identify and analyze potential attacks for risk assessment and elicitation of security requirements.

\section{ResEARCH Methodology}

This work is an exploratory study to form hypotheses about actual practices and knowledge sources for dealing with security requirements in real-world software projects. 
Three different research methods can typically help for such purposes: ethnography, interview, and survey.

Interviews and specially surveys may pose various biases due to questions phrasing, multiple-choice questions that are biased toward speci c answers, ambiguous questions, and data analysis methods. Ethnography studies may prevent many of such biases and provide a very detailed and accurate observation of the activities within teams and individual practitioners. However, accomplishing ethnography studies takes a long time while can target a limited number of $\mathrm{rms}$ or usually one team within one organization. In contrast, surveys are quick ways to collect uniform and massive amount of data from multiple and different rms and contexts.

\section{A. Structure of the Survey}

The survey in this study consists of 18 multiple choice questions. The list of questions and multiple choices are available in [1], and due to space limitations, we do not repeat questions in this paper. The questions focus on different aspects or methods related to security RE practices:

1.General and background: The rst group of questions gather general information about the type of the business domain, size of participating organizations, respondents' background and education, their role in the organization, and the use of security standards in the organization. We also asked about the regulation of security practices across the organization.

2. Security requirements: The second group of questions in the survey inquiries about the (security) RE practices such as modeling notations, documentation methods, and knowledge sources.

3. Security trade-off analysis: This group of questions investigates how security countermeasures are analyzed and whether security trade-offs are considered in selecting security mechanisms.

4. Security risk and attacks analysis: Finally, in the fourth group of questions, participants were asked about the risk assessment methods and consideration of common attacks in evaluating risk and eliciting security requirements.

\section{B. Data Collection Method}

In this survey, 400 software professionals from 237 Chinese and International organizations/ rms (located in China) were contacted, from which, 374 subjects participated in the survey. The survey was sent electronically to a network of software professionals, which had previous collaborations with the lead researcher in this project. Respondents' feedbacks were collected through a web-based survey.

\section{Participants}

Participants of the survey are software professionals of diverse roles from 237 Chinese organizations or branches of International companies in China. The majority of participating organizations belong to the IT industry ( $71 \%$ ), and the remained are involved in various sectors such as education, nance, banking, etc. $58 \%$ of the companies are private businesses, $15 \%$ are government-owned corporations, and the rest are multi-national rms, government agencies, and universities.

Table I lists roles of 374 participants in the survey. The majority of participants described their role as programmers (48\%), and in addition, 49 business and requirements analysts, 87 designers, and 63 project managers also responded to the survey. $64 \%$ of the participants have post-secondary education in computer science.

\section{Results And ObSERVATIONS}

The survey of security RE practices shows interesting and surprising security RE trends. This section provides the most signi cant observations as well as some correlation between different practices.

\section{A. Signi cant Findings}

Security education and training: good enough. $55 \%$ of total participants had received security training during postsecondary education, and $27 \%$ had received security training at their jobs. $17 \%$ of the participants never had any security training. Table 1 shows the status of security education and training with respect to their roles. IT and network specialists have received more security training than other groups, at their jobs and in total. Other groups with highest percentage of security training are requirements engineers and designers. Since, in total only $17 \%$ of participants have not received any security training, the population of participants have a suf cient security awareness to provide relevant feedback in the survey.

Adoption of security knowledge sources: moderately widespread. $48 \%$ of the respondents use security standards or guidelines. $42 \%$ stated that they themselves do not use any security standard or guideline, however, security standards are used in their organization. Among the participants who use security standards, $51 \%$ use Common Criteria and $19 \%$ use ISO 17799 Security Standard.

Figure 1 summarizes the use of different security knowledge sources. Although security knowledge portals such as NVD and CWE provide extensive and updated information about critical common vulnerabilities, $2 \%$ of participants were familiar with these portals and use them in security $\mathrm{RE}$ activities. The direct conclusion from this observation is that requirements analysts elicit security requirements isolated from known vulnerabilities. We hypothesize that these resources are not well known enough or they are not designed in a usable fashion. The vast amount of information provided in most of these knowledge sources are overwhelming, which makes nding relevant information to target domain, platforms, and technologies challenging.

The most popular sources of security knowledge are standards $(30 \%)$, books and design patterns $(30 \%)$, and 
Table I

SECURITY TRAINING STATUS FOR DIFFERENT ROLES

\begin{tabular}{|c|c|c|c|c|c|c|c|c|}
\hline & Total & $\begin{array}{l}\text { Training at } \\
\text { education }\end{array}$ & $\%$ & $\begin{array}{c}\text { Training at } \\
\text { job }\end{array}$ & $\%$ & $\begin{array}{l}\text { Not } \\
\text { trained }\end{array}$ & $\%$ & $\begin{array}{c}\text { Total trained } \\
\%\end{array}$ \\
\hline Requirements Engineer & 39 & 26 & $67 \%$ & 7 & $18 \%$ & 6 & $15 \%$ & $85 \%$ \\
\hline Business Analyst & 10 & 4 & $40 \%$ & 4 & $40 \%$ & 2 & $20 \%$ & $80 \%$ \\
\hline Designer & 87 & 48 & $55 \%$ & 27 & $31 \%$ & 12 & $14 \%$ & $86 \%$ \\
\hline Marketing & 21 & 9 & $43 \%$ & 6 & $29 \%$ & 6 & $29 \%$ & 71\% \\
\hline Software Developer/ Programmer & 258 & 143 & 55\% & 66 & $26 \%$ & 49 & $19 \%$ & 81\% \\
\hline IT/Network Specialist & 43 & 21 & $49 \%$ & 18 & $42 \%$ & 4 & $09 \%$ & 91\% \\
\hline Project Manager & 63 & 31 & $49 \%$ & 21 & $33 \%$ & 11 & $17 \%$ & $83 \%$ \\
\hline Security Analyst & 10 & 5 & $50 \%$ & 3 & $30 \%$ & 2 & 20\% & $80 \%$ \\
\hline
\end{tabular}

governmental security standards ( $12 \%)$. We hypothesize that the widespread use of standards usually stem from compliance requirements and audit processes. Patterns and best practices are favored by designers because these resources are reliable and easy to use.

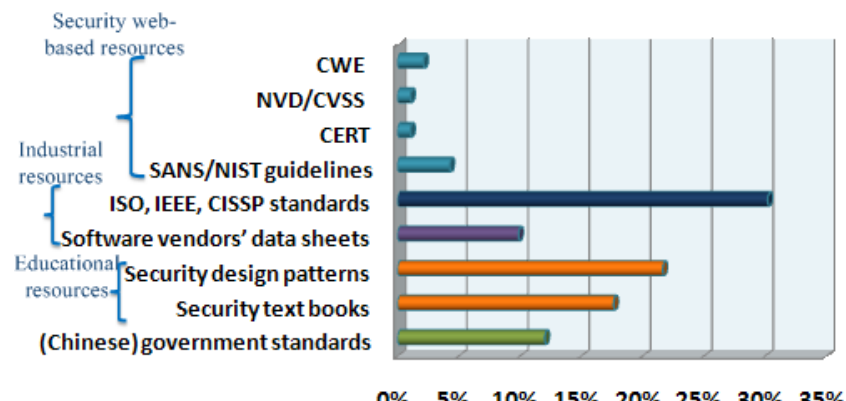

Figure 1. Security knowledge sources used by participants.

Security requirements elicitation and documentation: not a common practice. The survey results show that security requirements are often left undocumented, although analysts consider security issues at the early stages of the development. Only $9 \%$ of participants expressed that they explicitly document security requirements. $59 \%$ of participants expressed that they consider security requirements (implicitly), without documenting them explicitly. $31 \%$ expressed that they do not elicit security requirements at all, which con rms the existing belief that security requirements are not typically analyzed in enough detail and from early phases of the development life cycle [2].

This survey con rms the common belief that the software engineering community is beginning to realize that information and application security is also important for software whose primary function is not related to security [3]. For example, a decades ago the software engineering community started to move from the "penetrate and patch" approach to preventing the introduction of security vulnerabilities in the design and coding phases [4]. This survey shows security is not only considered at the coding and testing phases, and security requirements are being analyzed in earlier stages, often implicitly and sometimes explicitly.

Security is about trade-offs, but these trade-offs are usually ignored. The majority of participants $(88 \%)$ stated that security requirements and mechanisms may cause tradeoffs with other requirements and goals. In their opinion, security most often has con icts with performance, ef ciency, and some functionalities and features. $23 \%$ of participants do not consider multiple alternative security solutions for a given problem. From the rest of participants, $65 \%$ expressed that they only consider security level or nancial costs, or both to make trade-offs and select a solution among multiple alternative solutions. We concluded that trade-off analysis with full understanding of interactions among all requirements is not a common practice.

Consideration of common attacks: once bitten, twice shy. $72 \%$ of the participants stated that they document security attacks that have occurred, look for common attacks, or consult with security experts to prevent common attacks. The rest of the participants $(28 \%)$ do not consider attacks that have happened in the past as a source for eliciting security requirements. This shows that software professionals generally think about common attacks to elicit security requirements, thus, security RE frameworks need to provide methods to extract security requirements by relating them to common attacks (and vulnerabilities) and evaluating security countermeasures by the level of protection they provide against well-known attacks.

Security risk assessment: qualitative methods are favoured. Majority of the respondents (82\%) expressed that they analyze security risks. Respondents were given four options for evaluating security risks: 1- Qualitative labels of Low, Medium, High (33\% selected) 2- Ordinal scale of $1-9$ (49\% selected) 3 - Ordinal scale of 1-100 ( $8 \%$ selected) 4- Risks in terms of nancial costs (10\% selected). This observation indicates that evaluating risks in low-granular scales is preferred to detailed and numerical measures.

Conceptually, the ordinal scales of Low, Medium, High, $1-9$, and 1-100 are similar ways to assess risks; however, nearly half of the respondents state that the scale of 1-9 is more feasible and useful than the scale of 1-100. Only $8 \%$ of the respondents prefer the scale of 1-100.

Table II summarizes the relation of participants' role to their opinion about the most feasible and useful measures to evaluate security risks. Compared to other roles, designers and requirements analysts are more interested in assessing risks in qualitative measures that have a low granularity. Business and security analysts relatively show more interests 
Table II

USEFULNESS AND FEASIBILITY OF DIFFERENT SCALES FOR RISK EVALUATION FROM PARTICIPANTS' POINT OF VIEW

\begin{tabular}{|c|c|c|c|c|c|c|c|c|c|c|}
\hline & Total & $\begin{array}{l}\text { Low/Med } \\
\text { /High }\end{array}$ & $\%$ & $1-9$ & $\%$ & $1-100$ & $\%$ & $\begin{array}{c}\text { Financial } \\
\text { costs }\end{array}$ & $\%$ & \\
\hline Requirements Engineer & 39 & 15 & $38 \%$ & 17 & $44 \%$ & 2 & $5 \%$ & 5 & $13 \%$ & \\
\hline Business Analyst & 10 & 4 & $40 \%$ & 3 & $30 \%$ & 1 & $10 \%$ & 2 & $20 \%$ & $\sqrt{\text { Compared to other roles, }}$ \\
\hline Designer & 87 & 31 & $36 \%$ & 41 & $47 \%$ & 8 & $9 \%$ & 7 & $8 \%$ & have more interests in \\
\hline IT/Network Specialist & 43 & 16 & $37 \%$ & 15 & $35 \%$ & 7 & $16 \%$ & 5 & $12 \%$ & $\Rightarrow$ granular measures \\
\hline Project Manager & 63 & 24 & $38 \%$ & 28 & $44 \%$ & 6 & $10 \%$ & 5 & $8 \%$ & Avoid granular measures \\
\hline Security Analyst & 10 & 4 & $40 \%$ & 4 & $40 \%$ & 0 & $0 \%$ & 2 & $20 \%$ & \\
\hline
\end{tabular}

in using nancial costs than other groups. Project managers tend to avoid assessing risks in granular scales more than some other roles. This shows that a silver bullet method for risk assessment may not be favoured by all professional roles in software development.

The use of modeling notations: it is surprisingly widespread. $20 \%$ of the respondents document requirements in the natural language, and $54 \%$ structure requirements into tables. $57 \%$ also use some UML models to express the requirements, and $68 \%$ use non-UML models. The majority of respondents ( $73 \%$ ) expressed that they use standard or tailored modeling notations in their RE practices.

Half of the respondents use tailored modeling notations for dealing with security requirements, $36 \%$ use the same modeling notations that they use for analyzing other types of requirements, and $19 \%$ use a totally different modeling notation for analyzing security requirements.

A decade ago, security requirements and policies were seemed to be largely independent of system requirements and system models, and typically, system requirements and design are done rst, and security is added as an afterthought [2]. This survey shows that businesses have begun to realize the importance of considering security in modeling and analysis activities from earlier stages of the development.

\section{B. Correlation Analysis}

We pertained correlation analysis to discover relations and trends between different security RE practices. For example, is there any relation between the role of participants, such as requirements engineer, designers and security analyst, and taking more systematic approaches in dealing with security requirements? Does the risk evaluation method correlate with the diversity of factors that practitioners take into account for selecting countermeasures?

We apply Chi-square test to examine the statistical signi cance of the correlations. This test uses frequencies and proportions from a sample to test hypothesis about the corresponding population. Chi-square test works based on obtaining the observed frequency (i.e., the number of individuals in each category) and calculating the expected frequency (i.e., the hypothetical sample distribution that would be obtained if the sample proportions were according to the null hypothesis) [5]. The more the observed frequency differs from the expected frequency, the higher the probability of a signi cant correlation between elements being observed.

Security requirements elicitation and documentation. Unexpectedly, we discovered that there is no correlation between the roles of respondents and their approach to security requirements elicitation. Intuitively we expected to see that requirements engineers and security experts would elicit and document security requirements more explicitly than other roles do, but they tend to leave identifying and operationalizing security needs to developers. The survey results show that project managers are relatively more sensitive about eliciting and documenting security requirements compared to any other group. Project managers' interest in security may stem from their responsibility to assure security or may be due to the fact that managers are asked to take security into account from higher level management. While the survey clearly shows security is left to be achieved by the implementation team, relatively, developers show the least interest in security concerns.

The Chi-square test shows a signi cant correlation between explicit security requirements elicitation and consideration of common attacks. $50 \%$ of the participants who do not consider eliciting and documenting security requirements, do not consider common attacks to elicit security requirements either. $86 \%$ of the participants who document security requirements explicitly, tend to document or consider security attacks as well.

In order to explore why some analysts tailor common modeling techniques for security analysis (or use a different notation), we analyze the correlation between modeling notations and the consideration of common attacks in security RE. Our initial hypothesis was that analysts may need a tailored or different modeling technique to deal with security requirements if they analyze attacks and vulnerabilities.

However, the Chi-square test does not con rm our hypothesis strongly. There is a (non-signi cant) correlation between the subjects who look into attack and vulnerability data bases or ask security experts about common attacks and the type of modeling notation that they use when dealing with security requirements. $68 \%$ of participants who look into common attacks, tailor their modeling notations or use a totally different notation for analyzing security requirements. We hypothesize that attack and vulnerability analysis requires speci c security modeling elements which are not provided in common modeling techniques. 
Security risk assessment. The Chi-square test shows that there is a signi cant correlation between using qualitative risk assessment and considering multiple factors to select a solution among alternatives. We found out that people who assess risks qualitatively tend to consider more varieties of factors for selecting a solution. (56\% of respondents who use multiple criteria to decide on a security solution prefer qualitative risk assessment methods). We hypothesize that applying qualitative risk assessment methods require less detailed information about decision criteria, thus practitioners are able to take more diverse factors into consideration. The correlation analysis shows that there is also a statistically signi cant correlation between neglecting risk analysis and considering nancial costs as the only factor: people who do not assess risks tend to consider only nancial costs for selecting a solution.

$83 \%$ of respondents who expressed that they assess risk qualitatively, stated that evaluating risks by labels like Low, Medium, High or in the scale of 1-9 is more feasible than using nancial costs or more detailed scales. Respondents who prefer to assess risks quantitatively, in a later question, showed more interest in using Low, Medium, High and the scale of 1-9 (84\%) than the scale of 1-100 and nancial costs $(16 \%)$. This shows that the scale of 1-9 is understood both as a qualitative and quantitative method for assessing attacks impacts.

The Chi-square test shows a statistically signi cant correlation between the way the respondents elicit requirements preferences and the use of qualitative evaluation to assess the risks of attacks. Participants who rather using qualitative labels such as Low, Medium, High for evaluating risks, tend to rank requirements based on preferences instead of assigning numerical importance weights to each requirement.

\section{Threats to Validity}

This section discusses the biases and threats to validity of the survey results. We discuss how the impact of biases can be reduced.

\section{A. Survey Biases}

The quality of collected data in this surveys is subject to various validity issues. For example, factual questions sometimes elicit invalid or inaccurate answers. What respondents report as what they do may not accurately correspond to what they actually do. Small changes in wording of survey questions sometimes produce major changes in responses. The context in which a question is presented often has an impact on the way respondents answer questions.

Although this research is not prompted by prede ned hypotheses, the research did not start without any preconceived expectations. However it is possible that researchers have had strong values and commitment to the topic. For example, we expected that the study would show security requirements are not dealt in early phases of the development, security requirements are not usually gathered and communicated explicitly, and security trade-offs are invisible to the decision makers. Such hypotheses may have in uenced the survey, questions phrasing, and data analysis methods. Nevertheless, the survey results do not con rm some of the assumptions, which shows our initial hypothesis did not lead the survey toward biased conclusions.

\section{B. Bias Reduction Strategies}

Before lunching the survey, rst a pilot survey was conducted to examine the questions' wording and discover ambiguities in questions. The results of the pilot survey showed that some questions might be confusing, encouraging, or leading, which we corrected them in the actual survey. The pilot questionnaire was designed in English language. In order to avoid misinterpretations of questions by participants, in the nal survey, the questionnaire was translated to Chinese language.

The accuracy of responses is veri ed by having multiple respondents with similar roles in the same organization whose descriptions of the methods and practices should be similar. For example, in a rm with 8 respondents, 7 participants con rm that they consider common attacks, 6 respondents described their risk assessment method similarly, and 7 expressed that they do not use modeling notations. In total, although there exist few inconsistencies, the majority of responses converge toward similar directions.

In another approach to check the consistency of answers, a number of questions were repeated with different phrasing. For example, the risk assessment approach was investigated in more than one question. We compared the results with respect to these two questions, which shows there is a consistency between respondents' answers. For example, respondents who assess risks quantitatively, in later questions, showed more interest in using qualitative scales for evaluating the risks of attacks (84\%).

\section{RELATED WORK}

A few empirical surveys study the current RE practices [6], [7], [8], [9]. Similar eld studies and survey of current practices have been conducted speci cally on security RE methods. In [10], the requirements documents of 11 Swedish software projects have been analyzed and the study concluded that security requirements are inconsistent and poorly speci ed. Mouratidis et al. [11] conducted a survey to explore the rationale that governs implementation of information systems and network security expenditures. Another group of work focus on interactions of users with security mechanisms and human factors in developing secure solutions [12], [13].

There are industrial surveys that show the criticality of considering security measures in developing software systems, e.g., by estimating the costs of data breaches [14]. The study published by National Cyber Security Alliance [15] looks at security training within companies of variety 
of scale. WhiteHat website security statistics report [16] surveys the number of serious vulnerabilities in large, medium and small organizations.

Such eld studies and surveys of security practices do not uncover the methods and processes adopted by practitioners for eliciting, documenting, and analyzing security requirements. Studies such as [10] are useful to evaluate the performance of current security RE approaches, but these studies do not shed light on methods being used and their utility and usability.

\section{SUMmary AND CONCLUSIONS}

This paper describes an empirical study of security RE methods in real-world practice. In this study, different software development roles and positions in a number of Chinese and International rms were surveyed. We surveyed the approach in industry to security requirements elicitation, involvement of attacks and risk in the analysis, and the use of modeling, risk assessment, and quanti cation. We observed security requirements are not often explicitly elicited and documented in the early stages of the development, but instead are mostly considered during the implementation phase. The common approach about security requirements analysis has been the "Penetrate and Patch" scenario [4], but this survey shows the patching process has been replaced by secure software development at the coding stage.

In contrast to some existing assumptions about the lack of security training [17], in this survey, requirements engineers, product managers, software designers, and programmers stated that they have had security training and also use security knowledge sources and standards.

We conclude that security requirements are usually handled similar to other requirements and by ordinary software professionals. Trade-offs between security and other quality requirements are usually invisible to the software professionals. At the same time, most of the available body of software security knowledge is either unknown to the software professionals, and are not practical or are not intertwine with security RE activities.

Therefore, a practical security RE method needs to provide enough guidelines and methods for software professionals that are not security experts, and help them incorporate available security knowledge (from standards, check lists, web portals) into security requirements analysis activities. Security RE methods need to provide ways to identify security trade-offs and help decision stakeholders make an explicit and informed judgement.

A major limitation of this work is that the survey does not provide justi cation or explanations for the observations or correlations among practices. Assessing security risks by using qualitative value is more favoured by the practitioners, but we cannot conclude whether this is due to the unavailability and inaccuracy of detailed measures, lack of time and budget to acquire such measures, complexity of quantitative methods, or due to the lack of knowledge and training. Another limitations of this study is the population biases. The security was conducted in China within a number of Chinese and international rms, which may not represent the global view of the security RE methods.

\section{ACKNOWLEDGMENT}

Financial support from Natural Science and Engineering Research Council of Canada, National Natural Science Foundation of China grant number 60873064 and 90818026 is gratefully acknowledged.

\section{REFERENCES}

[1] G. Elahi and E. Yu, "Security Requirements Engineering Practices in the Wild: A Survey," available at ftp://ftp.cs.toronto.edu/csrg-technicalreports/610/SurveySuvrey Questions, 2011.

[2] P. T. Devanbu and S. G. Stubblebine, "Software engineering for security: a roadmap," in Proc. of ICSE'00. ACM, 2000, pp. 227-239.

[3] I. A. Tondel, M. G. Jaatun, and P. H. Meland, "Security requirements for the rest of us: A survey," IEEE Software, vol. 25, pp. 20-27, 2008.

[4] G. Mcgraw, "Testing for security during development: Why we should scrap penetrate-and-patch," IEEE Aerospace and Electronic Systems, vol. 13, pp. 13-15, 1998.

[5] F. J. Gravetter and L. B. Wallnau, Statistics for the Behavioral Sciences. Wadsworth Publishing, 2003.

[6] J. Aranda, S. Easterbrook, and G. Wilson, "Requirements in the wild: How small companies do it," Requirements Engineering,, vol. 0, pp. 39-48, 2007.

[7] L. Liu, H. Zhang, F. Peng, and W. Ma, "Understanding chinese characteristics of requirements engineering," Requirements Engineering, 2009.

[8] K. El Emam and N. H. Madhavji, "A eld study of requirements engineering practices in information systems development," in $R E$ ' 95,1995 , p. 68.

[9] D. Zowghi and D. Damian, "Field studies of requirements engineering in a multi-site software development organization: research in progress," in Proc. of Australian Workshop on Requirements Engineering, 2001.

[10] J. Wilander and J. Gustavsson, "Security requirements - a eld study of current practice," in E-Proc. of the Symposium on Requirements Engineering for Information Security, 2005.

[11] H. Mouratidis, H. Jahankhani, and M. Z. Nkhoma, "Management versus security specialists: an empirical study on security related perceptions," Inf. Manag. Comput. Security, vol. 16, no. 2, pp. 187-205, 2008.

[12] P. Dourish, E. Grinter, J. Delgado de la Flor, and M. Joseph, "Security in the wild: user strategies for managing security as an everyday, practical problem," Personal Ubiquitous Comput., vol. 8, no. 6, pp. 391-401, 2004.

[13] A. Adams and M. A. Sasse, "Users are not the enemy," Commun. ACM, vol. 42, no. 12, pp. 40-46, 1999.

[14] C. LeGrande and A. Hartman, "Does application security pay?" Mainstay Partners, 2010.

[15] "National small business study," National Cyber Security Alliance, 2010.

[16] "Whitehat security, whitehat website security statistic report, industry benchmarks," 2010.

[17] D. Firesmith, "Engineering security requirements," in Journal of Object Technology, vol. 2, no. 1, pp. 53-68, 2003. 